\title{
Seventeenth International Conference on Flow Injection Analysis
}

The $17^{\text {th }}$ International Conference on Flow Injection Analysis was held in Krakow, Poland, from 3 to 8 July, 2011. The conference was organized under the auspices of the Committee of Analytical Chemistry of the Polish Academy of Sciences and the Japanese Association for Flow Injection Analysis (JAFIA).

This was the first time that Poland had hosted this conference, which has been held regularly since 1989 . The organizing committee consisted of academic staff and $\mathrm{PhD}$ students from the Department of Analytical Chemistry of the Faculty of Chemistry, the Jagiellonian University. The Chairpersons of the Organizing Committee were Pawel Kościelniak and Joanna Kozak.

The organizers succeeded in drawing 141 participants from 24 countries to Krakow: in fact, the majority of scientists who over the years have contributed significantly to methodological and instrumental development as well as practical utilization of flow injection analysis were present at the conference. Invitations were accepted by, amongst others, Professors Jaromir Ruzicka and Elo Harald Hansen, regarded as the creators of this branch of analytical chemistry after they published an article entitled "Flow Injection Analysis. Part I. A New Concept of Fast Continuous Flow Analysis" in 1975. The conference was also attended by Professor Gary D. Christian, the originator of and tireless driving force behind the ICFIA conferences.

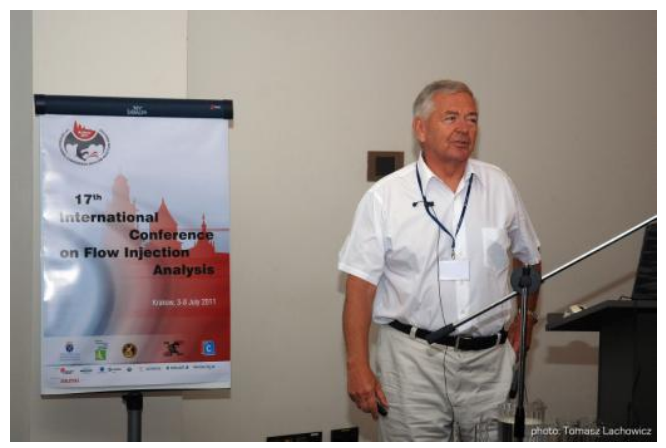

Another invited guest was Professor Marek Trojanowicz, the pioneer of flow analysis in Poland. The names of other lecturers, such as Purnendu Dasgupta, Jose Luis Burgera, Tadao Sakai, Jose Costa Lima, Victor Cerda, Manuel Miro and Jacobus Van Staden are well known not only to specialists dealing with flow analysis, but undoubtedly also to analytical chemists in general. The Special Guest of the conference was Professor Bogusław Buszewski, the Chair of the Polish Society of Chemistry.
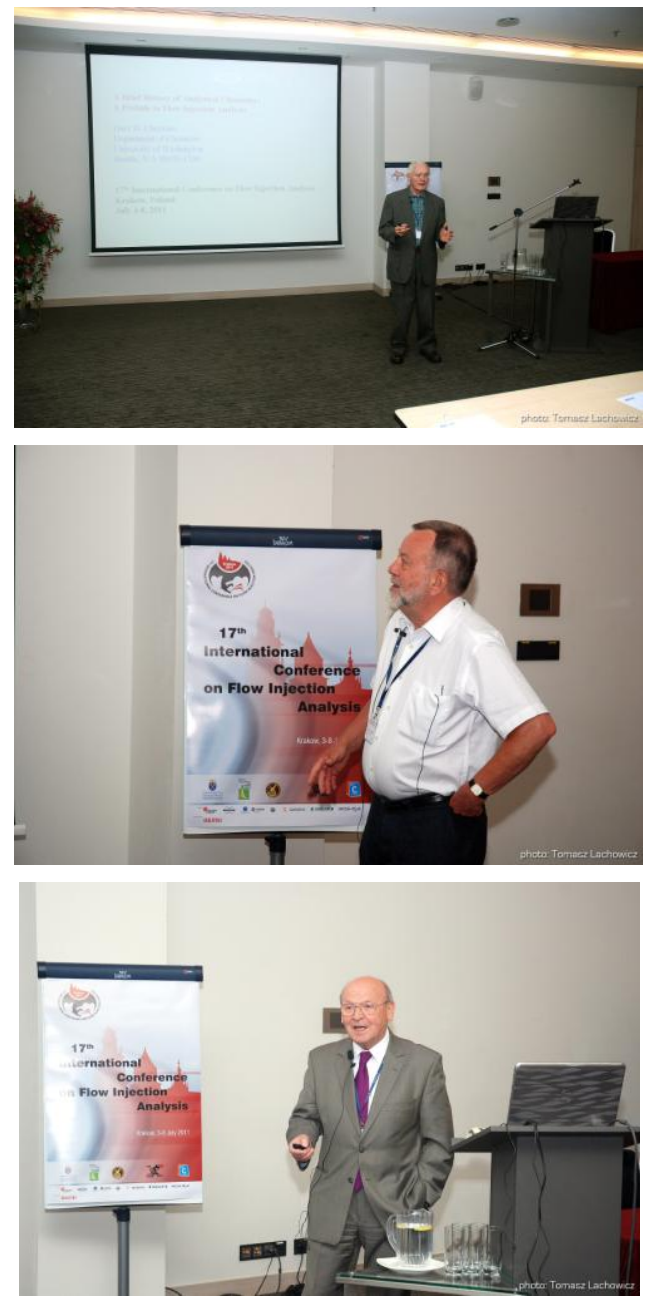

The lectures and oral presentations (54 contributions altogether) dealt with a variety of subjects. Among the topics covered were the history of flow injection analysis and the directions of its development ("Flow injection analysis - how it all started and later developed"). The place of flow injection analysis among other analytical techniques ("Where does open tubular liquid chromatography begin and flow injection analysis end?" as well as the outlook for its further development were debated ("Nanotechnology and flow systems", "Hybrid flow analysis systems and their application", "Combining quantum dots nanotechnology and flow analysis: prospects and applications"). Moreover, conceptions of modified flow techniques (e.g. cross injection 
analysis, stepwise injection analysis) and novel ideas in the field of sensors and detectors in flow analysis were put forward (e.g. "A dual-electrode flow sensor fabricated using tracketched microporous membranes", "Light emitting diodes in flow analysis", "Membrane electrostriction and its application in electrochemical detectors").

A significant majority of both the oral and the poster contributions (74 posters were presented) were devoted to analytical applications of flow systems in various fields of our life. Contributions relating to various aspects of environmental protection predominated. They included instrumental adaptation of systems (e.g. "Flow injection analysis: from beaker to microfluidics. Integrated and hyphenated sequential injection systems and their applications in oceanography", "Development of computer-controlled flow chemical analysis (CC-FCA) system and their use in environmental analysis") as well as their implementation in toxic substances control and monitoring (e.g. "Trace analysis of residue pharmaceuticals in environment as a new challenge for flow-injection analysis", "SIA and MSFIA determination of radiochemical isotopes in environmental samples" and "Determination of hydrogen ion concentration in acid rainwater by cyclic flow injection analysis"), or even the monitoring of ecological problems ("Use of flow injection analysis (FIA) for monitoring of hot mud flow and water quality at Porong area, East Java, Indonesia”).

Among the contributions relating to other aspects of practical application, those dealing with the implementation of flow systems in the field of food analysis were especially interesting (e.g. "Analysis of bisphenol a in milk by using an automatic fluorimetric sensor", "Monitoring of the pesticide carbaryl in table olives using a multicommutated-assembly" and "Automatic fluorimetric determination of thiabendazole residues in mushrooms"), as were papers concerning the determination of drugs and psychotropic substances for the purposes of toxicological and clinical research (e.g. "Lanthanide-Sensitized Luminescence as a promising tool in clinical analysis", "Determination of some drugs using WellsDawson molybdophosphate", "Contribution to automation for determination of drugs based on flow-through optosensing" and " $A$ rapid and automatic optosensor for the determination of ketorolac in pharmaceuticals and biological samples"). A range of interesting contributions concerning the increasingly popular issue of "green chemistry" should also be mentioned (e.g. "A reagent-free flow system for simultaneous analyses of dissolved carbon dioxide, sugar and color in carbonated drink" and "Strip test for iron using extract of guava leaf as natural reagent in combination of modern information technology devices: consideration with flow behavior approaches").

A special session was devoted entirely to the achievements of Polish scientists. Not only the conference venue - Poland but also the fact that the year 2011 had been declared the year of Marie Curie-Skłodowska (the $100^{\text {th }}$ anniversary of her Nobel Prize in Chemistry) made this a good occasion to broach this subject. A fascinating lecture dedicated to the life and scientific achievements of Marie Curie-Skłodowska was given by Bogusław Buszewski. A short review of academic centers in Poland dealing with flow analysis was presented by Pawel Kościelniak. In a further part of the session, lectures and oral presentations were given by representatives of the aforementioned centers, with Marek Trojanowicz at the forefront. The feature distinguishing these contributions from the others was the emphasis on particular fundamental analytical topics related to, e.g. calibration, multicomponent analysis and validation.

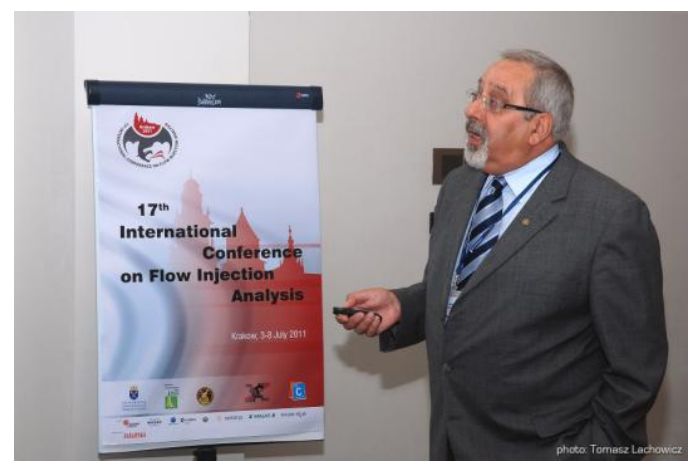

Thanks to the presence of many experienced chemists representing various views on the role and goals of flow techniques in analytical chemistry, discussions after presentations were quite often enthusiastic and spontaneous. Such discussions contributed additional academic and educational value, broadening, complementing and enriching the presentations. This constituted an excellent lesson for the many young researchers attending the conference. As the weather was appalling, the attendance at lectures and presentations was high beyond expectations and the conference debates undoubtedly made a great impression and were of scientific benefit.

It is not easy to concisely summarize the entire conference. Two important observations - perhaps entirely subjective spring to mind. Firstly, after years of rapid instrumental development in the field of flow analysis, generation of new 
flow techniques and designing of innovative constructions (e.g. multicommutation or lab-on-valve systems), a period of "calming down" seems to have descended slowly in this area and the focus has now shifted to ingenious and efficient application (often in combination) of instrumental concepts developed earlier to achieve specified analytical aims (e.g. determination of particular analytes, lowering detection limits or limiting the costs of analyses etc.). However, it should be noted that the above observation relates to flow systems on a macro scale. The issue of their miniaturization, so widely brought up during ICFIA conferences a few years ago, has become less popular at these meetings (as it seems to be a leading subject at other dedicated scientific meetings). The second observation, to some extent connected with the first one, is that against the background of the broad range of other flow techniques, the role of flow injection analysis as an independent technique seems to be decreasing - as its application appears to be less and less essential to achieve the aforementioned analytical aims. In other words, its characteristic and specific features are increasingly rarely employed in practice. In this context, the term "ICFIA" is slowly losing its connection with the actual state of affairs, although this situation does not seem to be threatening plans to organize further meetings under this banner, if for no other reason than out of respect for tradition.

The $17^{\text {th }}$ ICFIA conference was held in one of Krakow's modern hotels - the Hotel Park Inn by Radisson. Participants were cordially welcomed there and most of the various activities linked with the conference were held there: debates, poster presentations, presentations of apparatus and chemical accessories by companies, and conference lunches.

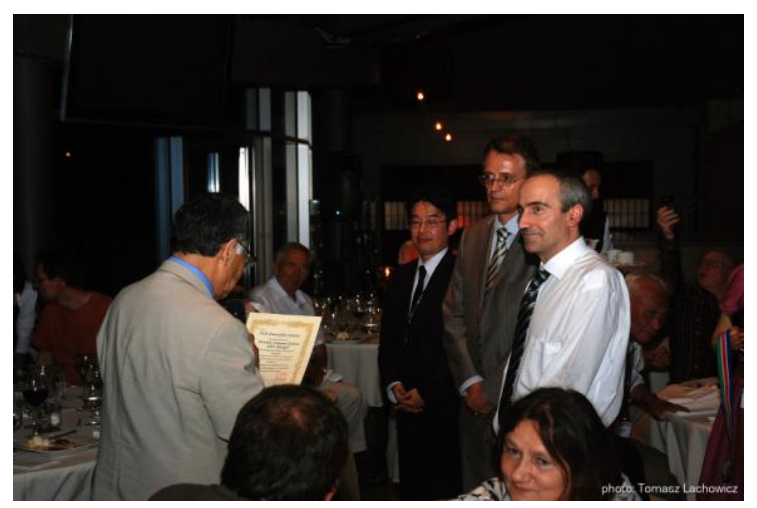

During the banquet, which was held in the Centre of Japanese Art and Technology "Manggha", the Chairman of JAFIA, Professor Tadao Sakai presented medals for scientific achievement in the field of flow analysis to: Spas Kolev from the Melbourne University, Antonio Rangel from the Portuguese Catholic University and Norio Teshima from the Aichi Institute of Technology. Moreover, two participants, Susana Vidigal from the Portuguese Catholic University and Rasha El Nashar from the German University in Cairo received equivalent awards (established by the organizers of the conference) for the best posters presented during the conference.
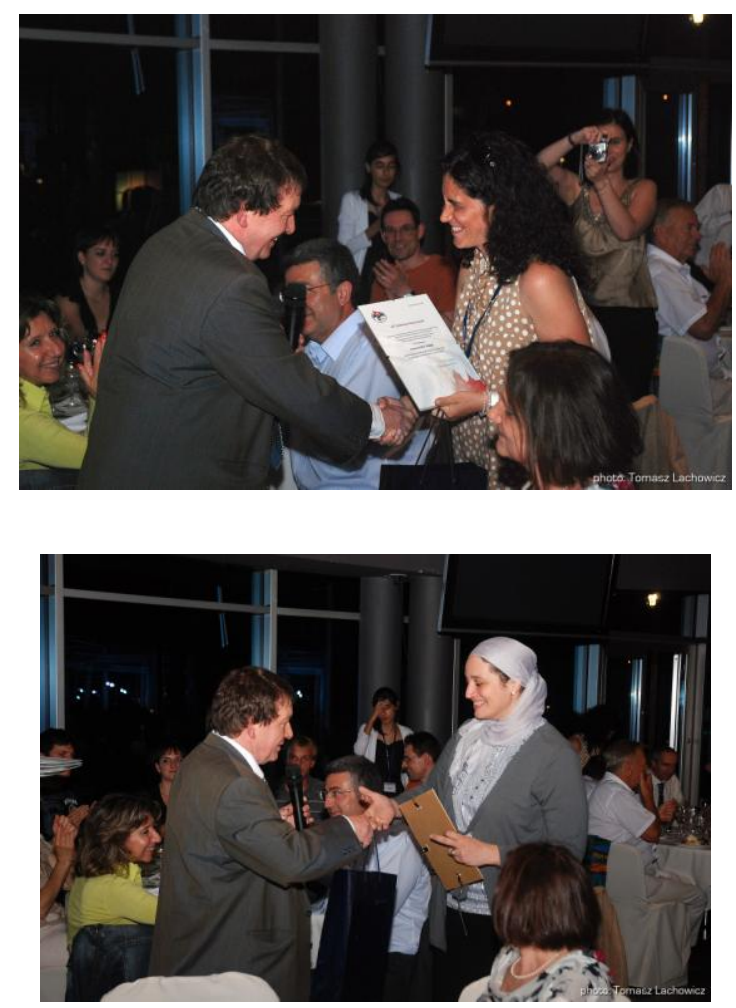

During their stay in Krakow, participants had an opportunity to do some sightseeing in our beautiful city. There was enough time to visit the Old Town with its splendid monuments, Collegium Maius, Wawel Hill or the vibrant and charming district of Kazimierz. A separate day was devoted to an excursion to Zakopane, a town situated at the foot of the Tatra Mountains, famous for its rich folklore and picturesque surroundings. During the excursion, participants had a chance to see the unique buildings of Chochołów and other places in the Podhale region. Unfortunately, it was not possible to fully appreciate the beauty spots of "old" Zakopane and the wonderful panorama of the Tatra Mountains from the top of Gubałówka Hill on account of the ceaseless rain. Hence, the participants accepted with relief an invitation to a highland inn, where they spent several hours enjoying a feast featuring regional cuisine, accompanied by folk music and dances. On the last day of the conference, after the closing ceremony, some 
participants took part in additional excursions to the "Wieliczka" Salt Mine or the Concentration Camp and Museum at Auschwitz Birkenau.

The organization of the conference in its final form would not have been possible without additional financial support. The main patron of the meeting was the Polish Academy of Sciences. Considerable assistance was also granted by the Organization for the Prohibition of Chemical Weapons, which paid the whole travel and attendance costs of five foreign participants. Among the companies that contributed to the organization of the conference, the following should be mentioned: Comesa Polska, Skalar Analytical BV, Sartorius Mechatronics Polska, WICOMESLAB, Perkin Elmer Polska and Bruker Polska. The media sponsor of the conference was the journal 'Analityka'. We would like to thank each of the sponsors once again.
The next $18^{\text {th }}$ International Conference on Flow Injection Analysis will be held in Porto, Portugal, in 2013 and will be hosted by Antonio Rangel from the Portuguese Catholic University. Moreover, people interested in flow analysis issues will have a chance to meet earlier, in 2012, in Thessaloniki, Greece, at the $12^{\text {th }}$ International Conference on Flow Analysis.

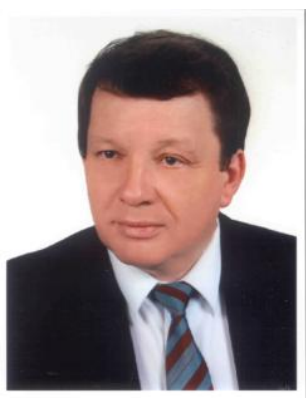

Paweł Kościelniak

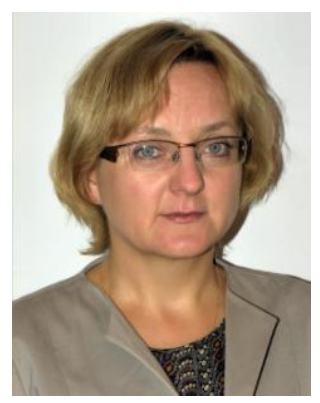

Joanna Kozak 